\title{
On the adjudication of conflicting claims: an experimental study
}

\author{
Carmen Herrero • Juan D. Moreno-Ternero • \\ Giovanni Ponti
}

Published online: 7 July 2009

(C) Springer-Verlag 2009

\section{Erratum to: Soc Choice Welf DOI 10.1007/s00355-009-0398-0}

During publication of this article, several mistakes were unfortunately made:

(1) The majority of the article note was deleted. The correct version should be as follows:

This paper is a much condensed version of Herrero et al. (2003), to which we refer for a more detailed discussion of the experimental results. We thank J. Agulló, F. Feri, J. Kovárik,

The online version of the original article can be found under doi:10.1007/s00355-009-0398-0.

\section{Herrero · G. Ponti $(\varangle)$}

Departamento de Fundamentos del Análisis Económico,

Universidad de Alicante, 03071 Alicante, Spain

e-mail: giuba@merlin.fae.ua.es

\section{Herrero}

IVIE, Valancia, Spain

e-mail: carmen.herrero@ua.es

J. D. Moreno-Ternero

Departamento de Teoría e Historia Económica, Universidad de Málaga, 29071 Málaga, Spain e-mail: jdmorenot@uma.es

J. D. Moreno-Ternero

Department of Economics, Universidad Pablo de Olavide, Seville, Spain

J. D. Moreno-Ternero

CORE, Université catholique de Louvain, Louvain-la-Neuve, Belgium

G. Ponti

Dipartimento Economia Istituzioni Territorio, Università di Ferrara, Ferrara, Italy 
D. López-Pintado, S. Mancinelli, R. Martínez, M. A. Meléndez, J. Mora, A. Moro, F. VegaRedondo, A. Villar and seminar participants at Brown, Caltech, Málaga, Milan, Rochester and Seville for helpful comments and suggestions. The paper was revised while J. MorenoTernero was visiting the University of Rochester. We would like to thank W. Thomson for his hospitality and valuable discussions. We also thank C. Belando, L. Garrido, V. Llorens and all people at the Laboratory for Theoretical and Experimental Economics (LaTEx) of the University of Alicante for their valuable help to run the experiments. Last, but not least, we are particularly grateful to the anonymous referees. Their detailed comments helped us to significantly improve the quality of the previous versions of this paper. Usual disclaimers apply.

Financial support from the Spanish Ministry of Science (BEC2001-0980, BEC20010535, CSD2006-16, ECO2008-03883, SEJ2005-04805, SEJ2007-62656), MIUR (PRIN 2007MCKEYA), Generalitat Valenciana (GV06/275), Junta de Andalucía (P06-SEJ-01645, P08-SEJ-04154) and Instituto Valenciano de Investigaciones Económicas (IVIE) is gratefully acknowledged.

(2) In the Tables 2, 3, 4 and 5 the values in the far right columns were given only in part. The correct versions are given here:

Table 2 Procedure $P_{1}$

\begin{tabular}{|c|c|c|c|c|c|c|c|c|c|c|c|}
\hline & $A$ & $B$ & $C$ & & $A$ & $B$ & $C$ & & $A$ & $B$ & $C$ \\
\hline \multirow{3}{*}{$A$} & -41.5 & -41.5 & -41.5 & & -41.5 & -41.5 & -41.5 & & -41.5 & -41.5 & -41.5 \\
\hline & -38.5 & -38.5 & -38.5 & $A$ & -38.5 & -38.5 & -38.5 & $A$ & -38.5 & -38.5 & -38.5 \\
\hline & 0 & 0 & 0 & & 0 & 0 & 0 & & 0 & 0 & 0 \\
\hline \multirow{3}{*}{$B$} & -41.5 & -41.5 & -41.5 & & -41.5 & -39.2 & -38.3 & & -41.5 & -38.3 & -38.3 \\
\hline & -38.5 & -38.5 & -38.5 & $B$ & -38.5 & -36.8 & -37.6 & $B$ & -38.5 & -37.6 & -37.6 \\
\hline & 0 & 0 & 0 & & 0 & -4 & -4.1 & & 0 & -4.1 & -4.1 \\
\hline \multirow{3}{*}{$C$} & -41.5 & -41.5 & -41.5 & & -41.5 & -38.3 & -38.3 & & -41.5 & -38.3 & -37.5 \\
\hline & -38.5 & -38.5 & -38.5 & $C$ & -38.5 & -37.6 & -37.6 & $C$ & -38.5 & -37.6 & -37.5 \\
\hline & 0 & 0 & 0 & & 0 & -4.1 & -4.1 & & 0 & -4.1 & -5 \\
\hline
\end{tabular}

Table 3 Procedure $P_{2}$

\begin{tabular}{|c|c|c|c|}
\hline & $A$ & $B$ & $C$ \\
\hline \multirow{3}{*}{$A$} & -41.5 & -39.2 & -39.2 \\
& -38.5 & -36.8 & -36.8 \\
& 0 & -4 & -4 \\
\hline \multirow{3}{*}{$B$} & -39.2 & -39.2 & -39.2 \\
& -36.8 & -36.8 & -36.8 \\
& -4 & -4 & -4 \\
\hline \multirow{3}{*}{$C$} & -39.2 & -39.2 & -39.2 \\
& -36.8 & -36.8 & -36.8 \\
& -4 & -4 & -4 \\
\hline
\end{tabular}

\begin{tabular}{|c|c|c|c|}
\hline & $A$ & $B$ & $C$ \\
\hline \multirow{4}{*}{$A$} & -39.2 & -39.2 & -39.2 \\
& -36.8 & -36.8 & -36.8 \\
& -4 & -4 & -4 \\
\hline \multirow{3}{*}{$B$} & -39.2 & -39.2 & -39.2 \\
& -36.8 & -36.8 & -36.8 \\
& -4 & -4 & -4 \\
\hline \multirow{3}{*}{$C$} & -39.2 & -39.2 & -39.2 \\
& -36.8 & -36.8 & -36.8 \\
& -4 & -4 & -4 \\
\hline
\end{tabular}

\begin{tabular}{|c|c|c|c|}
\hline & $A$ & $B$ & $C$ \\
\hline \multirow{4}{*}{$A$} & -39.2 & -39.2 & -39.2 \\
& -36.8 & -36.8 & -36.8 \\
& -4 & -4 & -4 \\
\hline \multirow{3}{*}{$B$} & -39.2 & -39.2 & -39.2 \\
& -36.8 & -36.8 & -36.8 \\
& -4 & -4 & -4 \\
\hline \multirow{3}{*}{$C$} & -39.2 & -39.2 & -37.5 \\
& -36.8 & -36.8 & -37.5 \\
& -4 & -4 & -5 \\
\hline
\end{tabular}


Table 4 Procedure $P_{2}$

\begin{tabular}{|c|c|c|c|}
\hline & $A$ & $B$ & $C$ \\
\hline \multirow{3}{*}{$A$} & -41.5 & -39.6 & -37.5 \\
& -38.5 & -36.6 & -37.5 \\
& 0 & -3.7 & -5 \\
\hline \multirow{3}{*}{$B$} & -39.6 & -39.6 & -37.5 \\
& -36.6 & -36.6 & -37.5 \\
& -3.7 & -3.7 & -5 \\
\hline \multirow{3}{*}{$C$} & -37.5 & -37.5 & -37.5 \\
& -37.5 & -37.5 & -37.5 \\
& -5 & -5 & -5 \\
\hline \multicolumn{3}{|c}{} \\
\end{tabular}

\begin{tabular}{|c|c|c|c|}
\hline & $A$ & $B$ & $C$ \\
\hline \multirow{4}{*}{$A$} & -39.6 & -39.6 & -37.5 \\
& -36.6 & -36.6 & -37.5 \\
& -3.7 & -3.7 & -5 \\
\hline \multirow{3}{*}{$B$} & -39.6 & -39.2 & -37.5 \\
& -36.6 & -36.8 & -37.5 \\
& -3.7 & -4 & -5 \\
\hline \multirow{3}{*}{$C$} & -37.5 & -37.5 & -37.5 \\
& -37.5 & -37.5 & -37.5 \\
& -5 & -5 & -5 \\
\hline
\end{tabular}

\begin{tabular}{|c|c|c|c|}
\hline & $A$ & $B$ & $C$ \\
\hline \multirow{4}{*}{$A$} & -37.5 & -37.5 & -37.5 \\
& -37.5 & -37.5 & -37.5 \\
& -5 & -5 & -5 \\
\hline \multirow{3}{*}{$B$} & -37.5 & -37.5 & -37.5 \\
& -37.5 & -37.5 & -37.5 \\
& -5 & -5 & -5 \\
\hline \multirow{3}{*}{$C$} & -37.5 & -37.5 & -37.5 \\
& -37.5 & -37.5 & -37.5 \\
& -5 & -5 & -5 \\
\hline
\end{tabular}

Table 5 Procedure $P_{0}$

\begin{tabular}{|c|c|c|c|}
\hline & $A$ & $B$ & $C$ \\
\hline \multirow{3}{*}{$A$} & -41.5 & -41.5 & -41.5 \\
& -38.5 & -47 & -47 \\
& 0 & 0 & 0 \\
\hline \multirow{3}{*}{$B$} & -50 & -39.2 & -50 \\
& -38.5 & -36.8 & -47 \\
& 0 & -6 & -6 \\
\hline \multirow{3}{*}{$C$} & -50 & -50 & -37.5 \\
& -38.5 & -47 & -37.5 \\
& 0 & -6 & -6 \\
\hline
\end{tabular}

\begin{tabular}{|c|c|c|c|}
\hline & $A$ & $B$ & $C$ \\
\hline \multirow{3}{*}{$A$} & -41.5 & -50 & -50 \\
& -38.5 & -36.8 & -47 \\
& -6 & -4 & -6 \\
\hline \multirow{3}{*}{$B$} & -39.2 & -39.2 & -39.2 \\
& -47 & -36.8 & -47 \\
& -4 & -4 & -4 \\
\hline \multirow{3}{*}{$C$} & -50 & -50 & -37.5 \\
& -47 & -36.8 & -37.5 \\
& -6 & -4 & -6 \\
\hline
\end{tabular}

\begin{tabular}{|c|c|c|c|}
\hline & $A$ & $B$ & $C$ \\
\hline \multirow{4}{*}{$A$} & -41.5 & -50 & -50 \\
& -38.5 & -47 & -37.5 \\
& -6 & -6 & -5 \\
\hline \multirow{3}{*}{$B$} & -50 & -39.2 & -50 \\
& -47 & -36.8 & -37.5 \\
& -6 & -6 & -5 \\
\hline \multirow{3}{*}{$C$} & -37.5 & -37.5 & -37.5 \\
& -47 & -47 & -37.5 \\
& -5 & -5 & -5 \\
\hline
\end{tabular}

(3) In the chapter "B.1.2 Instructions for an unframed session (Session 7)" footnote 32 is missing behind "SCREEN 3: THE FIRST GAME". This footnote should read:

32 This was the third game in Sessions 18, 19, 22 and 23, and the second game in Sessions 20, 21, 24 and 25.

The publisher apologises for any inconvenience caused by these mistakes. 